\title{
Guest Editorial: Special Issue in Memory of Mila Nikolova
}

\author{
Raymond H. Chan ${ }^{1} \cdot$ Albert Cohen $^{2}$ - Jalal Fadili ${ }^{3}$ - Alfred Hero ${ }^{4} \cdot$ Gabriele Steidl $^{5}$
}

Published online: 15 July 2020

C) Springer Science+Business Media, LLC, part of Springer Nature 2020

This special issue is in honor of our outstanding colleague Mila Nikolova (1962-2018), Research Director at Centre National de la Recherche Scientifique (CNRS), Microsoft Fellow, Simons Fellow, and IEEE Senior Member.

Starting her career as an engineer and science journalist in Sofia (Bulgaria), Mila obtained her PhD from the University Paris-Sud and worked at the Information Processing and Communication Laboratory at CNRS-ENST Paris before she joined the Ecole Normale Supérieure Cachan in 2003, where she raised to a Research Director at CNRS in 2009. Mila was awarded with the Michel Monpetit Prize from the French Academy of Science in 2010 "for the originality and depth of her research in mathematical image processing and in solving certain inverse problems."

Mila was a key figure of the international image processing community. She provided substantial results to the study of variational models with non-smooth data-fidelity terms and made important contributions to the development of a two-stage approach to restore images corrupted by blur and impulse noise which is still state of the art. She gave a systematic convergence rate analysis of half-quadratic minimization algorithms and achieved tightness results on the two-phase,

$凶$ Gabriele Steidl

steidl@math.tu-berlin.de

Raymond H. Chan

rchan.sci@ cityu.edu.hk

Albert Cohen

cohen@ann.jussieu.fr

Jalal Fadili

Jalal.Fadili@ensicaen.fr

Alfred Hero

hero@eecs.umich.edu

1 City University of Hong Kong, Hong Kong, China

2 Université Paris VI, Paris, France

3 CNRS-ENSICAEN-Université Caen, Caen, France

4 University of Michigan, Ann Arbor, MI, USA

5 Institut für Mathematik, TU Berlin, Strasse des 17. Juni 136, 10587 Berlin, Germany piecewise constant Mumford Shah model for image segmentation, to mention only a few of her results.

Mila was a person with extraordinary courage who spared no physical, material or logistical difficulties for multidisciplinary and multicultural collaborations. She will remain in her colleagues' minds through her important scientific work, her generosity, enthusiasm and mental strength.

This special issue features 14 invited articles of former collaborators and friends of Mila. The authors of these papers were greatly inspired by Mila's insights expressed succinctly in her published work as well as in the fertile technical discussions we have had with her during her lifetime.

Two of the contributions, namely A characterization of proximity operators (with R. Griboval) and A two-stage method for spectral-spatial classification of hyperspectral images (with R. H. Chan, K. Kan and R. Plemmons), were actually co-authored by Mila. These papers are of quite different content underlining the broad scientific interests of Mila. While the first paper characterizes proximity operators of convex or nonconvex penalties extending well-known results of Moreau, the second one proposes a novel two-stage method for the classification of hyperspectral images.

Many mathematical imaging problems are posed as nonconvex optimization problems, and several contributions are related to such models. So the paper New insights on the optimality conditions of the $\ell_{2}-\ell_{0}$ minimization problem (E. Soubies, L. Blanc-Féraud, G. Aubert) analyzes necessary optimality conditions for the 0-regularized least-squares minimization problem that are the roots of the plethora of algorithms to cope with this NP-hard problem. In $O n$ non-convex total variation regularizers for convex signal denoising (I. Selesnick, A. Lanza, S. Morigi, F. Sgallari), the authors propose a non-convex TV regularizer using concepts from convex analysis that unifies, generalizes and improves upon a certain class of regularizers. The authors of Bregman Itoh-Abe methods for sparse optimisation (M. Benning, E. S. Riis, C.-B.Schönlieb) construct optimization methods for variational regularization problems based on discretizing the inverse scale space flow with discrete gradient methods for non-smooth, non-convex optimization and give conver- 
gence guarantees in a Clarke subdifferential framework. In nonconvex optimization, one has usually to fight with local minima. When the problem is formulated in terms of maximizing the likelihood function under a statistical model for the measurements, one can construct a statistical test that a local maximum is in fact the global one, useful for testing global convergence of iterative local optimizers. Mila often argued from such statistical point of view. In the paper Testing that a local optimum of the likelihood is globally optimum using reparameterized embeddings (J. LeBlanc, B. Thelen, A. Hero), a one-sided test is introduced that applies to the case where the statistical model is a member of the generalized location family of probability distributions, a condition often satisfied in imaging and other inverse problems.

Two contributions take quite different, closer looks at the total variation regularization. In Crouzeix-Raviart approximation of the total variation on simplicial meshes (A. Chambolle, T. Pock), the authors construct an adaptive implementation of a Crouzeix-Raviart-based discretization of the total variation, which has the property of approximating from below the total variation, with metrication errors only depending on the local curvature. The paper Tensor factorization with total variation and Tikhonov regularization for low-rank tensor completion in imaging data (X.-L. Lin, M. K. Ng, X.-L. Zhao) incorporates a hybrid regularization combining total variation and Tikhonov regularization into low-tubal-rank tensor factorization model for low-rank tensor completion.

A new proximal interior point algorithm which is able to handle convex optimization models involving the sum of a Lipschitz differentiable term and a possibly nonsmooth one is considered in A proximal interior point algorithm with applications to image processing (E. Chouzenoux, M.-C. Corbineau, J.-Ch. Pesquet). The authors of Stable backward diffusion models that minimise convex energies ( $\mathrm{L}$. Bergerhoff, M. Cárdenas, J. Weickert, M. Welk) establish a backward diffusion model which implements a smart stabilization approach that can be used in combination with certain numerical schemes to handle the ill-posed and highly unstable inverse problem of backward diffusion. Backward diffusion processes appear naturally in image enhancement and deblurring applications.

Image segmentation is a topic of the papers Multi-layer joint segmentation using MRF and graph cuts (N. Lermé, S. Le Hégarat-Mascle, F. Malgouyres, M. Lachaize) and
Unsupervised assignment flow: label learning on feature manifolds by spatially regularized geometric assignment (A. Zern, M. Zisler, S. Petra, Ch. Schnörr). The first paper segments several images at once which allows for an increase of the information. The authors of the second paper introduce an unsupervised assignment flow that couples the assignment flow for supervised image labeling with Riemannian gradient flows for label evolution on feature manifolds, thus encompassing extensions of state-of-the-art clustering approaches to manifold-valued data.

The challenging problem of fine structure detection with applications to bituminous surfacing crack recovery is examined in A nonlocal Laplacian-based model for bituminous surfacing crack recovery and its MPI implementation (N. Debroux, C. Le Guyader, L. A. Vese). Based on the observation that such structures can be suitably modeled by a sequence of smooth functions whose Hessian matrices blow up in the perpendicular direction to the crack, while their gradient is null, the new model also handles the natural dense and highly oscillatory texture exhibited by the images.

Finally, the work Correcting the side effects of ADC filtering in MR image reconstruction (C. Lazarus, M. März, P. A. Weiss) investigates the role of the filters implemented on Analog-to-Digital Converters for the reconstruction of magnetic resonance images. When the distance between consecutive samples is larger than Shannon's limit, severe degradation of the reconstructed images may appear. A mathematical model that allows to mitigate such filtering effects is proposed which opens new challenges in sampling theory.

All manuscripts underwent the usual peer reviewing of JMIV, and care has been taken to avoid any conflict of interest: No author was involved in the decision process. We hope that this special issue mirrors the high mathematical standard Mila imposes on her own work.

The Guest Editors,

Raymond H. Chan, Albert Cohen, Jalal Fadili, Alfred Hero and Gabriele Steidl

Publisher's Note Springer Nature remains neutral with regard to jurisdictional claims in published maps and institutional affiliations. 